\title{
Consumo de cromo e estado nutricional de universitários em Sobral, Ceará, Brasil
}

\author{
Chrome consumption and nutritional status of university colleges \\ in Sobral, Ceará, Brazil
}

Ana Gleyciane de Lima Rocha ${ }^{1}$, Jorge Luis Pereira Cavalcante ${ }^{2}$

Rocha AGL, Cavalcante JLP. Consumo de cromo e estado nutricional de universitários em Sobral, Ceará, Brasil / Chrome consumption and nutritional status of university colleges in Sobral, Ceará, Brazil. Rev Med (São Paulo). 2019 set.-out.;98(5):298-303.

RESUMO: O objetivo desta pesquisa foi avaliar o estado nutricional e o consumo alimentar de cromo de acadêmicos de uma instituição de ensino superior. Foi aplicado um Questionário de Frequência Alimentar e realizou-se antropometria em acadêmicos de um centro universitário no interior do Ceará. Participaram 125 alunos matriculados no curso de Nutrição dos quais 19 eram homens $(15,2 \%)$ e 106 eram mulheres $(84,8 \%)$ com média de faixa etária de 24 anos. As variáveis antropométricas foram peso, altura, circunferência da cintura e quadril. Como resultados, as seguintes médias foram obtidas: $63,41 \mathrm{~kg}$ de peso, $1,64 \mathrm{~m}$ de altura, 30,08 $\%$ do índice de adiposidade corporal e $1,12 \mathrm{~m}^{2} / \mathrm{kg}$ do índice de conicidade no público feminino; e $57,28 \mathrm{~kg}$ de peso, $1,59 \mathrm{~m}$ de altura, $21,76 \%$ do índice de adiposidade corporal e $1,08 \mathrm{~m}^{2} / \mathrm{kg}$ do índice de conicidade no público masculino. Através do Índice de Massa Corpórea, foi certificado que $67,2 \%$ dos participantes apresentaram sobrepeso/obesidade, $28,8 \%$ eutrofia e $4,0 \%$ baixo peso. Em relação ao consumo alimentar, averiguou-se a frequência em que os indivíduos ingeriam alimentos com baixo, médio e alto teor de cromo, constatando frequência de 1 a 4 vezes por dia. Com isso, todos os acadêmicos apresentaram ingestão diária desse mineral, ressaltando-se que $62 \%$ dos alimentos ingeridos concentraram-se no consumo de altas quantidades de carboidratos refinados, o que não é favorável em termos de estado nutricional. Concluiu-se que o consumo dietético diário de cromo aponta que os acadêmicos estão ingerindo porções suficientes do mineral, evitando possível deficiência, apesar do sobrepeso e obesidade estarem em dominância. Isso poderia ser um dos fatores que elevaria o risco de diabetes mellitus tipo II.

Descritores: Consumo de alimentos; Cromo; Estudantes; Estado nutricional; Diabetes mellitus; Estudantes de ciências da saúde.

\begin{abstract}
The objective of this research was to evaluate the nutritional status and food consumption of chromium of academics of a higher education institution. A Food Frequency Questionnaire was applied and anthropometry was carried out in academics from a university center in the interior of Ceará. 125 students enrolled in the Nutrition course were enrolled, of which 19 were men (15.2\%) and 106 were women $(84.8 \%)$, with a mean age of 24 years. The anthropometric variables were weight, height, waist and hip circumference. As results, the following averages were obtained: $63.41 \mathrm{~kg}$ of body weight, $1.64 \mathrm{~ms}$ of height, $30.08 \%$ of body fat index and $1.12 \mathrm{~m}^{2} / \mathrm{kg}$ of female conicity index; and $57.28 \mathrm{~kg}$ of body weight, $1.59 \mathrm{~m}$ of height, $21.76 \%$ of body fat index and $1.08 \mathrm{~m}^{2} / \mathrm{kg}$ of male conicity index. Through the Body Mass Index, it was certified that $67.2 \%$ of the participants were overweight / obese, $28.8 \%$ eutrophic and $4.0 \%$ underweight. In relation to food consumption, the frequency of eating foods with low, medium and high levels of chromium was observed, with a frequency of 1 to 4 times a day. Thus, all the students presented daily intake of this mineral, noting that $62 \%$ of the food intake was concentrated in the consumption of high amounts of refined carbohydrates, which is not favorable in terms of nutritional status. It was concluded that the daily dietary intake of chromium indicates that the students are ingesting sufficient portions of the mineral, avoiding possible deficiency, despite being overweight and obesity in dominance. This could be one of the factors that would increase the risk of type II diabetes mellitus.
\end{abstract}

Keywords: Food consumption; Chromium; Students; Nutritional status; Diabetes mellitus; Students, health occupations.

\footnotetext{
1. Nutricionista, Bacharela em Nutrição pelo Centro Universitário Inta (UNINTA). ORCID: https://orcid.org/0000-0003-3864-0049. E-mail: anagleyciane@ gmail.com.

2. Nutricionista, Bacharel em Nutrição pela Universidade Estadual do Ceará (UECE), Especialista em Dietoterapia (UNIFOR), Mestre em Ciência e Tecnologia de Alimentos pela Universidade Federal do Ceará (UFC), Doutorando em Nutrição pela Universidad Internacional Iberoamericana (UNINI México) e Docente do Curso de Bacharelado em Nutrição do Centro Universitário Inta (UNINTA). ORCID: https://orcid.org/0000-0001-9168-9517. E-mail: jorgeluispcavalcante@uninta.edu.br.

Endereço para correspondência: Jorge Luis Pereira Cavalcante. Centro Universitário UNINTA, Coordenação do Curso de Bacharelado em Nutrição. Rua Coronel Antônio Rodrigues Magalhães, nº 359. Bairro Dom Expedito - Sobral, CE, Brasil. CEP 62.050-100. E-mail: jorgeluispcavalcante@uninta.edu.br.
} 


\section{INTRODUÇÃO}

diabetes mellitus tipo II (DM) é uma doença crônica caracterizada por perda progressiva de secreção insulínica combinada com resistência à insulina, e defeitos na secreção e ação da insulina e na regulação da produção de glicose no fígado ${ }^{1}$. O predomínio da doença tem se alastrado e a tendência é que englobe o aumento das faixas etárias mais jovens ${ }^{2}$. $\mathrm{O}$ número de pessoas com a doença é bastante significativo e está associado a vários fatores incluindo o aumento da urbanização e industrialização, fatores genéticos e ambientais como o sedentarismo, obesidade e alimentação ${ }^{3-4}$.

$\mathrm{O}$ aumento de doenças crônicas como a diabetes mellitus tipo II pode não ser uma consequência da idade, mas sim do resultado de consumo alimentar inadequado, podendo estar associado à deficiência de nutrientes com a sensibilidade à insulina. Sendo assim, dentre os nutrientes presentes, o mineral cromo tem despertado o interesse de vários pesquisadores para resposta da essencialidade desse mineral na ação da insulinas .

Diante disso, tem-se sugerido que o mineral age como cofator da insulina, onde há um aperfeiçoamento da ação do hormônio no transporte eficiente da glicose para a célula $^{6-7}$. Sendo assim, foi proposto que o mineral aumenta a fluidez da membrana celular mediando a sinalização de forma a facilitar a ligação da insulina com o seu receptor ${ }^{8}$.

Pressupõe-se que a deficiência do mineral na dieta possa contribuir para o desenvolvimento de resistência insulínica e/ou diabetes mellitus tipo $\mathrm{II}^{9-11}$. Assim, sugerese que o estado nutricional do indivíduo com deficiência atribua a diminuição da tolerância à glicose e, em consequência disso, leve ao quadro clínico ${ }^{12}$. No entanto, essa questão não está confirmada pela falta de dados da ingestão de cromo $\left(\mathrm{Cr}^{3+}\right)$ na dieta e pela inexistência de indicadores que identifiquem o estado nutricional em relação ao mineral ${ }^{8,11-13}$.

O objetivo deste estudo foi avaliar o estado nutricional e o consumo alimentar de cromo de acadêmicos de uma Instituição de Ensino Superior de Sobral - CE.

\section{CAUSUÍSTICA E MÉTODOS}

O presente trabalho tratou-se de uma pesquisa de campo, quantitativa, exploratória e descritiva realizada no Centro Universitário INTA (UNINTA), Sobral - CE, entre fevereiro e maio de 2018 com alunos do Curso de Bacharelado em Nutrição. Os participantes do estudo foram aqueles com idade acima de 18 anos, ambos os sexos e regularmente matriculados na instituição. Foram excluídos os acadêmicos com licença médica; os sob transferência, trancamento ou abandono; aqueles em uso de polivitamínicos; e alunas em período gestacional. Como a investigação foi realizada no período da noite, dos
182 alunos matriculados neste turno, 125 compuseram a amostra (19 homens - 15,2\%, 106 mulheres - 84,8\%; e com idade variando de 19 a 39 anos - média de 24 anos). Foi considerado um IC $=95 \%$ e erro amostral de 0,05 .

Após assinatura do Termo de Consentimento Livre e Esclarecido (TCLE), foi realizada em cada aluno os seguintes procedimentos: aferição da altura utilizando um estadiômetro portátil; peso obtido através de balança digital; circunferências da cintura e quadril com o auxílio de uma fita inelástica. Seguiram-se padrões de referência ${ }^{14}$ para os valores obtidos do Índice de Massa Corpórea (IMC); para o Índice de Adiposidade Corporal (IAC) ${ }^{15}$; e para o Î́ndice de Conicidade (IC) ${ }^{16}$.

Cada aluno também respondeu um Questionário de Frequência Alimentar (QFA) que mensura quantitativamente a frequência de consumo de cada produto da lista. Os dados do questionário serviram para discriminação da ingestão de cromo pelos estudantes. A Tabela 1 contém os alimentos com baixa, média e alta quantidade do mineral.

As planilhas Microsoft Excel 2016 foram utilizadas para a elaboração de estatística básica descritiva (média, desvio padrão e porcentagens) e dos dados da antropometria.

Tabela 1 - Quantidade de cromo nos principais alimentos

\begin{tabular}{|c|c|c|}
\hline $\begin{array}{l}\text { Baixa quantidade } \\
\text { (<1 } \mu \text { g por porção) }\end{array}$ & $\begin{array}{l}\text { Média } \\
\text { quantidade } \\
\text { (de } 1 \text { a } 3 \mu \mathrm{g} \text { por } \\
\text { porção) } \\
\end{array}$ & $\begin{array}{l}\text { Alta quantidade } \\
\text { (>3 } \boldsymbol{\mu g} \text { por } \\
\text { porção) }\end{array}$ \\
\hline Abacate & Grãos integrais & Condimentos \\
\hline Melão/Melancia & Cereais integrais & Fígado \\
\hline Mamão & $\begin{array}{l}\text { Leguminosas/ } \\
\text { Feijões }\end{array}$ & Vísceras/Miúdos \\
\hline Leites fermentados & Nozes & $\begin{array}{l}\text { Levedo de } \\
\text { cerveja/Levedura }\end{array}$ \\
\hline Açúcares refinados & Cogumelos & Ostras \\
\hline $\begin{array}{l}\text { Farinha de trigo } \\
\text { refinada }\end{array}$ & $\begin{array}{l}\text { Ameixas e } \\
\text { passas }\end{array}$ & $\begin{array}{l}\text { Tubérculos } \\
\text { (batatas) }\end{array}$ \\
\hline Cereais refinados & Aspargos & Brócolis \\
\hline & Cerveja, vinho & Alho \\
\hline Iogurte & Queijos & Suco de uva \\
\hline Requeijão & Ovos & Vegetais/Legumes \\
\hline \multirow[t]{7}{*}{ Manteiga/Margarina } & Farelo de trigo & \\
\hline & $\begin{array}{l}\text { Carnes (boi, } \\
\text { frango, peixe) }\end{array}$ & \\
\hline & Óleo de milho & \\
\hline & Moluscos & \\
\hline & Suco de laranja & \\
\hline & Banana & \\
\hline & Maçã & \\
\hline
\end{tabular}

Os alimentos e a quantidade de cromo em microgramas $(\mu \mathrm{g})$ foram retirados de informações contidas nas obras dos pesquisadores: Silva, Pires e Cozzolino ${ }^{10}$, Mussoi ${ }^{16}$, Anderson et al. ${ }^{17}$, Alvarado-Gámez et al. ${ }^{18}$, Nix ${ }^{19}$, Wardlaw e Smith ${ }^{20}$. 
A presente pesquisa foi submetida ao Comitê de Ética em Pesquisa do UNINTA, cujo parecer foi aprovado sob o número 2.496.423 e CAAE 82423917.0.0000.8133.

\section{RESULTADOS}

A Tabela 2 expõe as médias dos valores adquiridos na antropometria dos participantes da pesquisa.

Tabela 2 - Valores das médias \pm desvio-padrão (DP) de peso, altura, Índice de Massa Corpórea (IMC), Circunferência da Cintura (CC), Circunferência do Quadril (CQ), Índice de Adiposidade Corporal (IAC) e Índice de Conicidade (IC) obtidos na realização da antropometria dos estudantes de Nutrição $(n=125)$ conforme o gênero. Sobral, 2018

\begin{tabular}{c|c|c|c|c|c|c|c|c}
\hline ALUNOS & \multicolumn{1}{c}{ IDADE $^{1}$} & \multicolumn{1}{c}{ PESO $^{2}$} & ALTURA $^{3}$ & IMC $^{4}$ & CC $^{5}$ & CQ $^{6}$ & IAC $^{7}$ & IC $^{8}$ \\
\hline $\begin{array}{l}\text { Mulheres } \\
(\mathrm{n}=106)\end{array}$ & $24 \pm 3,84$ & $63,41 \pm 11,98$ & $1,64 \pm 0,09$ & $23,63 \pm 3,46$ & $76 \pm 8,26$ & $98 \pm 9,31$ & $30,08 \pm 4,19$ & $1,12 \pm 0,04$ \\
$\begin{array}{l}\text { Homens } \\
(\mathrm{n}=19)\end{array}$ & $25 \pm 4,31$ & $57,28 \pm 10,35$ & $1,59 \pm 0,08$ & $22,57 \pm 3,17$ & $71 \pm 6,48$ & $96 \pm 6,80$ & $21,76 \pm 3,70$ & $1,08 \pm 0,03$ \\
\hline
\end{tabular}

Legenda: ${ }^{1}$ em anos; ${ }^{2}$ em kg; ${ }^{3}$ em cm $;{ }^{4} \mathrm{em} \mathrm{kg} / \mathrm{m}^{2} ;{ }^{5} \mathrm{em} \mathrm{cm} ;{ }^{6} \mathrm{em} \mathrm{cm} ;{ }^{7} \mathrm{em} \% ;{ }^{8} \mathrm{em} \mathrm{m} / \mathrm{kg}$.

Segundo os valores obtidos no cálculo do IC, foi verificada a forma de cilindros em média dos participantes, embora individualmente parte deles apresente a forma de cones duplos. Na utilização da fórmula do IAC, foram verificadas médias acima dos parâmetros considerados para eutrofia, mostrando prevalência de sobrepeso em ambos os sexos. Vale ressaltar que esses métodos antropométricos são mais eficazes e mais aceitos do que o IMC ou Índice de Quetelet (IQ), se tratando de quantificação e centralização de gordura.

De acordo com as circunferências, o risco de complicações metabólicas associadas à obesidade foi constatado apenas em parte dos participantes avaliados, prevalecendo um valor médio de CC de $76 \mathrm{~cm}$ para mulheres e $71 \mathrm{~cm}$ para os homens, e média da CQ de 98 $\mathrm{cm}$ para o sexo feminino e $96 \mathrm{~cm}$ para o sexo masculino.

$\mathrm{Na}$ avaliação do estado nutricional através do IMC, certificou-se que $67,2 \%$ apresentaram sobrepeso/obesidade, $28,8 \%$ dos participantes apresentaram eutrofia e 4,0\% manifestaram baixo peso, sendo o público feminino mais predominante.

Em relação ao consumo alimentar, averiguou-se a frequência em que os indivíduos ingeriam os alimentos com a presença de cromo por meio do QFA, onde foi observado que parte dos indivíduos procuravam manter uma alimentação saudável com ingestão de frutas, legumes, vegetais e carnes. Porém, muitos preferiam os carboidratos refinados como massas e cereais, bem como pouco consumo de produtos integrais.

A análise da ingestão diária e semanal de cromo apresentou frequência de 1 a 10 vezes, como mostram as Tabelas 3 e 4 . Ao analisar a ingestão de cromo em itens com baixo teor do mineral na alimentação dos estudantes, constatou-se frequência de 1 a 4 vezes por dia em um total de 92 alunos (73,6\%), tendo preferência a fruta, açúcar, pães, leite, iogurte e margarina/manteiga. A frequência semanal desse mesmo total de acadêmicos variou de 1 a 10 vezes, tendo em vista a escolha de insumos como frutas, macarrão, açúcar e pizza/panqueca.

Tabela 3 - Frequência diária do consumo de alimentos contendo baixa, média e alta quantidade de cromo

\begin{tabular}{|c|c|c|c|c|}
\hline \multirow{2}{*}{$\begin{array}{l}\text { Alimentos com frequência } \\
\text { diária }\end{array}$} & \multicolumn{2}{|c|}{1 a 2 vezes } & \multicolumn{2}{|c|}{3 a 4 vezes } \\
\hline & Número & $\%$ & Número & $\%$ \\
\hline \multicolumn{5}{|l|}{$\begin{array}{l}\text { Baixa quantidade de cromo } \\
(<1 \mu \mathrm{g} \text { por porção) }\end{array}$} \\
\hline Abacate & 0 & 0,0 & 0 & 0,0 \\
\hline Melão/Melancia & 3 & 2,4 & 0 & 0,0 \\
\hline Mamão & 13 & 10,4 & 0 & 0,0 \\
\hline Açúcar & 9 & 7,2 & 0 & 0,0 \\
\hline Macarrão & 0 & 0,0 & 0 & 0,0 \\
\hline Pães & 18 & 14,4 & 0 & 0,0 \\
\hline Pizza/Panqueca & 2 & 1,6 & 0 & 0,0 \\
\hline Leite integral ou desnatado & 18 & 14,4 & 1 & 0,8 \\
\hline Iogurte natural ou com frutas & 11 & 8,8 & 0 & 0,0 \\
\hline Manteiga/Margarina & 17 & 13,6 & 0 & 0,0 \\
\hline \multicolumn{5}{|l|}{$\begin{array}{l}\text { Média quantidade de cromo } \\
\text { (1 a } 2 \mu \text { g por porção) }\end{array}$} \\
\hline Leguminosas (feijões) & 18 & 14,4 & 2 & 1,6 \\
\hline Cerveja, vinho & 0 & 0,0 & 0 & 0,0 \\
\hline Queijos & 10 & 8,0 & 0 & 0,0 \\
\hline Gema de ovos & 10 & 8,0 & 1 & 0,8 \\
\hline Boi & 6 & 4,8 & 1 & 0,8 \\
\hline Frango & 5 & 4,0 & 0 & 0,0 \\
\hline Peixes & 2 & 1,6 & 0 & 0,0 \\
\hline Óleos/Azeite & 26 & 20,8 & 0 & 0,0 \\
\hline Suco de laranja & 7 & 5,6 & 0 & 0,0 \\
\hline Banana & 15 & 12,0 & 1 & 0,8 \\
\hline Maçã & 8 & 6,4 & 0 & 0,0 \\
\hline \multicolumn{5}{|l|}{$\begin{array}{l}\text { Alta quantidade de cromo ( }>2 \\
\mu \mathrm{g} \text { por porção) }\end{array}$} \\
\hline Condimentos & 13 & 10,4 & 0 & 0,0 \\
\hline Vísceras, miúdos & 6 & 4,8 & 0 & 0,0 \\
\hline Tubérculos (batatas) & 6 & 4,8 & 1 & 0,8 \\
\hline Brócolis/Couve-flor/Repolho & 13 & 10,4 & 3 & 2,4 \\
\hline $\begin{array}{l}\text { Abobrinha/Berinjela/Chuchu/ } \\
\text { Pepino/Tomate/Cenoura }\end{array}$ & 10 & 8,0 & 0 & 0,0 \\
\hline
\end{tabular}


Tabela 4 - Frequência semanal do consumo de alimentos contendo baixa, média e alta quantidade de cromo

\begin{tabular}{|c|c|c|c|c|c|c|c|c|c|c|}
\hline \multirow{2}{*}{ Alimentos com frequência semanal } & \multicolumn{2}{|c|}{1 a 2 vezes } & \multicolumn{2}{|c|}{3 a 4 vezes } & \multicolumn{2}{|c|}{5 a 6 vezes } & \multicolumn{2}{|c|}{7 a 8 vezes } & \multicolumn{2}{|c|}{$\begin{array}{l}9 \text { a } 10 \\
\text { vezes }\end{array}$} \\
\hline & $\mathrm{N}$ & $\%$ & $\mathrm{~N}$ & $\%$ & $\mathrm{~N}$ & $\%$ & $\mathrm{~N}$ & $\%$ & $\mathrm{~N}$ & $\%$ \\
\hline \multicolumn{11}{|l|}{ Baixa quantidade de cromo ( $<1 \mu \mathrm{g}$ por porção) } \\
\hline Abacate & 26 & 20,8 & 16 & 12,8 & 5 & 4,0 & 2 & 1,6 & 0 & 0,0 \\
\hline Melão, melancia & 32 & 25,6 & 13 & 10,4 & 4 & 3,2 & 1 & 0,8 & 0 & 0,0 \\
\hline Mamão & 34 & 27,2 & 27 & 21,6 & 11 & 8,8 & 4 & 3,2 & 1 & 0,8 \\
\hline Açúcar & 23 & 18,4 & 24 & 19,2 & 12 & 9,6 & 11 & 8,8 & 3 & 2,4 \\
\hline Macarrão & 29 & 23,2 & 18 & 14,4 & 1 & 0,8 & 2 & 1,6 & 0 & 0,0 \\
\hline Pães & 14 & 11,2 & 36 & 28,8 & 15 & 12,0 & 23 & 18,4 & 0 & 0,0 \\
\hline Pizza, panqueca & 31 & 24,8 & 5 & 4,0 & 1 & 0,8 & 19 & 15,2 & 1 & 0,8 \\
\hline Leite integral, desnatado & 14 & 11,2 & 37 & 29,6 & 24 & 19,2 & 8 & 6,4 & 7 & 5,6 \\
\hline Iogurte & 42 & 33,6 & 18 & 14,4 & 11 & 8,8 & 0 & 0,0 & 0 & 0,0 \\
\hline Manteiga, margarina & 18 & 14,4 & 24 & 19,2 & 0 & 0,0 & 19 & 15,2 & 1 & 0,8 \\
\hline \multicolumn{11}{|l|}{ Média quantidade de cromo ( 1 a $2 \mu \mathrm{g}$ por porção) } \\
\hline Leguminosas & 24 & 19,2 & 25 & 20,0 & 28 & 22,4 & 7 & 5,6 & 6 & 4,8 \\
\hline Cerveja, vinho & 14 & 11,2 & 1 & 0,8 & 0 & 0,0 & 0 & 0,0 & 0 & 0,0 \\
\hline Queijos & 28 & 22,4 & 39 & 31,2 & 16 & 12,8 & 1 & 0,8 & 1 & 0,8 \\
\hline Gema de ovos & 16 & 12,8 & 57 & 45,6 & 14 & 11,2 & 8 & 6,4 & 5 & 5,0 \\
\hline Boi & 44 & 35,2 & 38 & 30,4 & 16 & 12,8 & 1 & 0,8 & 1 & 0,8 \\
\hline Frango & 25 & 20,0 & 49 & 39,2 & 26 & 20,8 & 9 & 7,2 & 2 & 1,6 \\
\hline Peixes & 56 & 44,8 & 5 & 5,0 & 1 & 0,8 & 0 & 0,0 & 0 & 0,0 \\
\hline Óleos, azeite & 23 & 18,4 & 22 & 17,6 & 19 & 15,2 & 8 & 6,4 & 2 & 1,6 \\
\hline Suco de laranja & 25 & 20,0 & 23 & 18,4 & 34 & 27,2 & 9 & 7,2 & 9 & 7,2 \\
\hline Banana & 12 & 9,6 & 41 & 32,8 & 28 & 22,4 & 12 & 9,6 & 8 & 6,4 \\
\hline \multicolumn{11}{|l|}{ Alta quantidade de cromo ( $>2 \mu \mathrm{g}$ por porção) } \\
\hline Condimentos & 18 & 14,4 & 12 & 9,6 & 14 & 11,2 & 2 & 1,6 & 2 & 1,6 \\
\hline Vísceras, miúdos & 44 & 35,2 & 39 & 31,2 & 16 & 12,8 & 1 & 0,8 & 1 & 0,8 \\
\hline Tubérculos (batatas) & 33 & 26,4 & 16 & 12,8 & 0 & 0,0 & 1 & 0,8 & 0 & 0,0 \\
\hline Brócolis, couve-flor, repolho & 19 & 15,2 & 33 & 26,4 & 4 & 3,2 & 1 & 0,8 & 1 & 0,8 \\
\hline $\begin{array}{l}\text { Abobrinha, berinjela, chuchu, pepino, tomate, } \\
\text { cenoura }\end{array}$ & 34 & 27,2 & 17 & 13,6 & 11 & 8,8 & 2 & 1,6 & 1 & 0,8 \\
\hline
\end{tabular}

Fonte: Autoria própria (2018).

Averiguando a frequência da ingestão do mineral em itens com média quantidade, em $89,6 \%$ do total de alunos, foram citados alimentos como leguminosas, incluindo feijões, óleos refinados e azeite, sendo observada maior ingestão de carne de boi, peixe, maçã, queijos, ovos, carne de boi, frango, banana e suco de laranja.

Dos alimentos com alto teor do íon cromo investigados no questionário foram destacados os 
condimentos, brócolis/couve/repolho e vegetais/legumes, onde $41,6 \%$ do total de estudantes marcaram frequência diária de 1 a 4 vezes. Na frequência semanal, o uso de itens como vísceras/miúdos, tubérculos e vegetais/legumes foram verificados de 1 a 2 vezes com maior frequência, assim como os itens anteriores.

Muitos alimentos apresentaram altas porcentagens de frequência de consumo de cromo nos grupos contendo baixa e média quantidade desse mineral. Porém, há presença de uma porcentagem de ingestão suficiente de alimentos contendo alto teor do mineral, levando majoritariamente a justificativa de que os estudantes poderiam estar ingerindo certas quantidades ao longo do dia, favorecendo a uma possível adequação do nutriente.

\section{DISCUSSÃO}

Não há muitos estudos atuais sobre ingestão alimentar de cromo. Uma pesquisa realizada no Rio de Janeiro por Silva mostrou análise da ingestão dietética de cromo em um período de três meses em 14 atletas de basquetebol, do sexo masculino com faixa etária entre 20 e 37 anos. $\mathrm{O}$ autor utilizou o inquérito do Recordatório 24 horas (R24) para o estudo, sendo efetuado 3 vezes por semana. Ele observou que todos os atletas consumiam quantidades de cromo acima de $35 \mu \mathrm{g} / \mathrm{dia}$, resultando na ingestão diária de $90 \mu \mathrm{g}$, em média. No entanto, cabe ressaltar que as recomendações nutricionais não são destinadas a atletas e também não há determinação de limites máximos de ingestão ${ }^{21}$.

Pei et al. ${ }^{22}$ utilizaram alimentos e complementos alimentares enriquecidos com cromo. Os pesquisadores avaliaram o efeito do leite em pó enriquecido com 200 $\mu \mathrm{g}$ de cloreto de cromo, em estudo duplo-cego controlado em 60 pacientes durante 16 semanas. Houve uma redução significativa na glicemia e insulinemia de jejum, especialmente em pacientes do sexo masculino.

A falta de novos estudos sobre a ingestão do nutriente na alimentação pode ser justificada pelo avanço das indústrias farmacêuticas. O suplemento picolinato de cromo é um produto desenvolvido por essas indústrias, apostando nos benefícios do mesmo para a saúde da população com DM tipo II ou não ${ }^{23}$. Em relevância, uma pesquisa feita na Bolívia mostrou a eficiência do suplemento de picolinato de cromo, podendo ajudar a aumentar a massa corporal magra, reduzir a porcentagem de gordura no corpo e consequentemente, reduzir o peso corporal total quando o indivíduo está em prática de atividade física ${ }^{24}$.

No entanto, também há controvérsias no estudo randomizado, controlado por placebo e levedura de cromo realizado por Kleefstra et al..$^{25}$ em uma população ocidental para investigar os efeitos no controle glicêmico e na resistência à insulina, durante um período de seis meses. $\mathrm{O}$ estudo foi realizado em 57 indivíduos, onde um grupo de 28 sujeitos ficou como placebo e outro grupo de 29 sujeitos com levedura de cromo $(400 \mu \mathrm{g} / \mathrm{dia})$. Os resultados não demonstraram evidências de que a suplementação fosse eficaz para o controle glicêmico em indivíduos com DM, mostrando um ligeiro aumento na HbA1c (7,0\%). Sendo assim, a ingestão de cromo através da alimentação se torna mais segura, uma vez que indivíduos sem patologias levem em consideração a ingestão diária escolhendo alimentos favoráveis.

Diante disso, um estudo com adolescentes verificou que a nutrição é essencial quando se abordam as mudanças fisiológicas associadas ao decorrer da idade. Foi demonstrado um certo grau de contribuição do estado nutricional dos indivíduos, em relação às necessidades fisiológicas, por nutrientes para manutenção das funções adequadas do organismo ${ }^{26-27}$. Com isso, embora todos os acadêmicos apresentassem ingestão diária de cromo, ressalta-se que $62 \%$ da quantidade de alimentos ingeridos concentraram-se no consumo de altas quantidades de carboidratos, o que não é favorável em termos de estado nutricional, absorção e biodisponibilidade do cromo, podendo justificar o alto índice de sobrepeso e obesidade ${ }^{28}$.

\section{CONCLUSÕES}

Conclui-se que a ingestão alimentar dos acadêmicos contendo cromo foi observada com grande frequência. Porém, ainda há alta ingestão de alimentos ricos em carboidratos refinados, onde a inserção suficiente do mineral na alimentação ou o uso de suplementação seria ineficiente, já que esses fatores desfavorecem a biodisponibilidade do nutriente. Com isso, levanta-se a hipótese de que a deficiência do mineral poderia reduzir a tolerância à glicose e levar um indivíduo ao quadro clínico de diabetes, com o passar do tempo. Nesse sentido, poderiam ser iniciadas ações de educação em saúde para evitar a incidência da doença.

Participação dos autores: Ana Gleyciane de Lima Rocha participou da concepção, coleta de dados e escrita do manuscrito. Jorge Luis Pereira Cavalcante participou da concepção, escrita do manuscrito e revisão crítica do manuscrito.

\section{REFERÊNCIAS}

1. Oliveira JEP, Foss-Freitas MC, Junior RMM, Vencio S. Diretrizes da Sociedade Brasileira de Diabetes 2017/2018. São Paulo: Clannad; 2017. Disponível em: https://www.diabetes. org.br/profissionais/images/2017/diretrizes/diretrizessbd-2017-2018.pdf.

2. Tonetto IFA, Baptista MHB, Gomides DS, Pace AE. Quality of life of people with diabetes mellitus. Rev Esc Enferm USP. 2019;53:e03424. doi: http://dx.doi.org/10.1590/s1980220x2018002803424. 
3. Sartorelli DS, Franco LJ. Tendências do diabetes mellitus no Brasil: o papel da transição nutricional. Cad Saude Pública. 2003;19(Sup. 1):S29-S36. doi: http://dx.doi.org/10.1590/ S0102-311X2003000700004.

4. Ortiz MCA, Zanetti ML. Levantamento dos fatores de risco para diabetes mellitus tipo 2 em uma instituição de ensino superior. Rev Latino-Am Enfermagem. 2001;9(3):58-63. doi: http://dx.doi.org/10.1590/S0104-11692001000300009.

5. Pereira LO, Francischi RP, Lancha Jr AH. Obesidade: hábitos nutricionais, sedentarismo e resistência à insulina. Arq Bras Endocrinol Metab. 2003;47(2):111-27. doi: http://dx.doi. org/10.1590/S0004-27302003000200003.

6. Carvalheira JBC, Zecchin HG, Saad MJA. Vias de Sinalização da Insulina. Arq Bras Endocrinol Metab. 2002;46(4):419-25. doi: http://dx.doi.org/10.1590/S0004-27302002000400013.

7. Fonseca LA, Girardi FM, Coelho CS, Barioni G, Rangel VB, Gonçalves RC. Influence of chromium supplementation on energy metabolism in horses used in policing activity. Arq Bras Med Vet Zootec. 2011;63(5):1175-80. doi: http://dx.doi. org/10.1590/S0102-09352011000500019.

8. Falcão LEM. Suplementação de cromo associado ao exercício físico. RBNE. 2016;10(57):343-9. Disponível em: http:// www.rbne.com.br/index.php/rbne/article/view/647/558.

9. Gomes MR, Rogero MM, Tirapegui J. Considerações sobre cromo, insulina e exercício físico. Rev Bras Med Esporte. 2005;11(5):262-6. doi: http://dx.doi.org/10.1590/S151786922005000500003 .

10. Silva AGH, Pires LV, Cozzolino SMF. Cromo. In: Cozzolino SMF. In: Biodisponibilidade de nutrientes. Barueri: Manole; 2015.

11. Simões ALR. Intervenção do cromo na relação da insulina e exercício físico. São Paulo: Associação Brasileira de Medicina Biomolecular; 2011.

12. Lukaski HC. Magnesium, zinc, and chromium nutriture and physical activity. Am J Clin Nutr. 2000;72(Supl 2):585S-593S. doi: https://doi.org/10.1093/ajen/72.2.585S.

13. Chehade JM, Sheikh-Ali M, Mooradian AD. The role of micronutrients in managing diabetes. Diabetes Spectrum. 2009;22:214-8. doi: https://doi.org/10.2337/diaspect.22.4.214.

14. Associação Brasileira para o Estudo da Obesidade e da Síndrome Metabólica - ABESO. Diretrizes Brasileiras de Obesidade. São Paulo: ABESO; 2016. Disponível em: http:// www.abeso.org.br/uploads/downloads/92/57fccc403e 5 da. pdf.

15. Bergman RN, Stefanovski D, Buchanan TA, Sumner AE, Reynolds JC, Sebring N et al. A better index of body adiposity. Obesity (Silver Spring). 2011;19(5):1083-9. doi: https://doi. org/10.1038/oby.2011.38

16. Mussoi TD. Avaliação nutricional na prática clínica: da gestação ao envelhecimento. Rio de Janeiro: Guanabara Koogan; 2014.

17. Anderson RA, Bryden NA, Polansky MM. Dietary chromium intake. Freely chosen diets, institutional diets, and individual foods. Biol Trace Elem Res. 1992;32(1):117-21. https://link. springer.com/article/10.1007/BF02784595.

18. Alvarado-Gámez A, Blanco-Sáenz R, Mora-Morales E. El cromo como elemento esencial en los humanos. Rev Costarric Cienc Méd. 2002;23(1-2):55-68. Disponível em: http:// www.scielo.sa.cr/scielo.php?script=sci_arttext\&pid=S025329482002000100006\&lng=en.

19. Nix S. Minerais. In: Nix S. Nutrição básica \& dietoterapia. Rio de Janeiro: Elsevier; 2010.

20. Wardlaw GM, Smith AM. Água e minerais. In: Wardlaw GM, Smith AM. Nutrição contemporânea. Porto Alegre: Artemed/ McGrawHill; 2013.

21. Silva LFM. Teor de cromo em alimentos e ingestão dietética de cromo por atletas de basquetebol [tese]. Campos dos Goytacazes: UENF; 2014. Disponível em: http://uenf. br/posgraduacao/producao-vegetal/wp-content/uploads/ sites/10/2015/01/TESE-Luiz-Fernando-Miranda-da-Silva. pdf.

22. Pei D, Hsieh CH, Hung YJ, Li CH, Kuo SW. The influence of chromium chloride-containing milk to glycemic control of patients with type 2 diabetes mellitus: a randomized, doubleblind, placebo-controlled trial. Metabolism. 2006;55(77):9237. doi: https://doi.org/10.1016/j.metabol.2006.02.021.

23. Jaramillo RAM. Importancia del cromo en el organismo de personas con diabetes tipo II. Rev Tec U. 2007;5(5):3-7. Disponível em: http://www.revistasbolivianas.org.bo/pdf/ rtc/v5n5/v5n5a02.pdf.

24. Muñoz EL, Colman EL, Blanco LL. El efecto del cromo en el síndrome metabólico [monografia]. Madrid: Universidad Complutense de Madrid; 2016. Disponível em: http://147.96.70.122/Web/TFG/TFG/Memoria/LUCIA\%20 LOPEZ\%20BLANCO.pdf.

25. Kleefstra N, Houweling ST, Bakker SJL, Verhoeven S, Gans ROB, Meyboom DE, et al. Chromium treatment has no effect in patients with type 2 diabetes in an Western Population: a randomized, double-blind, placebo-controlled trial. Diabetes Care. 2007;30(5):1092-6. doi: https://doi.org/10.2337/dc062192.

26. Sales-Peres SHC, Goya S, Sant' anna RMF, Silva HM, SalesPeres AC, SILVA RPR, Lauris JRP, et al. Prevalência de sobrepeso e obesidade e fatores associados em adolescentes na região centro-oeste do estado de São Paulo (SP, Brasil). Ciênc Saúde Coletiva. 2010;15(Supl 2):3175-84. doi: http:// dx.doi.org/10.1590/S1413-81232010000800022.

27. Toimil RFSL, Ferreira SRG. Nutrição no diabetes melitus. In: Silva SMCS, Mura JDP. Tratado de alimentação, nutrição e dietoterapia. São Paulo: Roca; 2010.

28. Paiva AN. Suplementação oral com picolinato de cromo em pacientes com diabetes tipo 2: um ensaio clínico randomizado [tese]. Natal: UFRN; 2015. doi: https://repositorio.ufrn.br/ handle/123456789/20372.

Recebido: 21.05 .19

Aceito: 21.08 .19 